\title{
Mathematical \& Physical Modelling of a Quadrotor UAV
}

\author{
MOAD IDRISSI *
}

Faculty of Computing, Engineering and the Built Environment

Birmingham City University

Birmingham, UK

Moad.Idrissi@mail.bcu.ac.uk

MOHAMMAD SALAMI

Faculty of Computing, Engineering and the Built Environment

Birmingham City University

Birmingham, UK

Mohammad.salami@bcu.ac.uk

FAWAZ ANNAZ

Faculty of Computing, Engineering and the Built Environment

Birmingham City University

Birmingham, UK

fawaz.annaz@bcu.ac.uk

Unmanned aerial vehicles (UAVs) are now becoming a major topic of interest due to their flying capabilities attracting researchers who are working within various application. Quadrotors in particular are one of main types of UAVs that are now currently studied, where some of the main focuses are positional and attitude tracking. Currently, verifying these systems in simulation is generally processed through MATLAB/Simulink where the dynamics are thoroughly analyzed. In this paper, the results attained from the mathematical dynamics implemented in Simulink will be justified using ADAMS environment. This software was purposely developed to accurately model the dynamics of mechanical systems in 3D without considering any equations of motion. SolidWorks is used to design the quadrotor frame that satisfies the properties of the proposed system in Simulink. Setting the control inputs as angular velocity of each motor will generate a relative thrust in order for the vehicle to achieve motion. Finally, the dynamic behavior on ADAMS and Simulink are compared as the control inputs are identically applied, which has revealed a marginal difference between the resultant motions.

Additional Keywords and Phrases: UAV, Quadrotor, Physical Modelling, Msc ADAMs

* Corresponding author contact email: Moad.Idrissi@mail.bcu.ac.uk 


\section{INTRODUCTION}

Over the past years, unmanned aerial vehicles (UAVs) have received a great interest from researchers and hobbyists worldwide due to their great potential applications. Specifically, Vertical take-off and landing (VTOLs) vehicles have caught more interest due to their significant orientation capabilities as well as the implementation in many applications such as rescue missions, surveillance, military operations, explorations and many more [1]. A specific VTOL type of vehicle called the Quadrotor has recently emerged as a popular platform for many researchers due to the ease of development and maintenance [2]. Additionally, these vehicles have the capabilities of quick maneuverability, hovering and agile motions in confined areas [3].

Quadrotors in general consist of an ' $X$ ' frame that has four motors with propellers placed at each end respectively, the motion of the vehicle is described by increasing or decreasing the speed of the individual motors. However, this carries the fact of under actuation making it incapable of achieving all six degrees of freedom simultaneously [4]. Therefore, many authors have designed various control techniques $[5,6,7]$ to achieve autonomous flying, in which numerical data is calculated to present the dynamics.

In terms of analyzing the motions of the quadrotor, many authors including those in $[8,9,10]$ have presented the performances based on Simulation tools such as Simulink. In fact, the software is relied on greatly to present results in which authors have monumentally trusted. This introduces a drawback in the experimental/practical development where small errors can be easily overlooked, resulting in unexpected outcomes compared to simulation. Researchers have attempted to overcome this issue by using a physical modelling software such as Msc ADAMS, which was developed by the United States MDI Corporation for static, kinematic and dynamic analysis of a virtual mechanical system [10].

For instance, the authors in [11] have proposed a method of using the co-simulation feature in Simulink to create control inputs, which are then passed onto ADAMS to present the dynamics of the vehicle. This was initiated to achieve full UAV motion while implementing Sliding Mode Proportional Integral Derivative (SMCPID) control. Following a presentation of the theory behind SMC-PID control, the authors revealed that a joint simulation between both software has accurately verified the performance of the proposed control algorithm.

The authors in [12] have recently analyzed a tilt-rotor UAV theoretically in MATLAB which was compared against ADAMS environment that consists of the mechanical geometry. It has been determined that as the rotors tilt, the moment of inertia changes and effects the attitude response of the vehicle. By using ADAMS to accurately model the proposed system, the authors concluded that the structural changes due to the motor rotation makes it challenging to mathematically model these behaviors in comparison to a traditional quadrotor. In [13], a research into V-tail UAVs has been undertaken to justify the vehicle performance using the cosimulation feature between ADAMS and MATLAB. The author mentions that very little research has been conducted into verifying the mathematical model. Thus, ADAMS environment is used to accurately present the dynamic behavior with identical physical parameters to the Simulink model. Although there is research carried out to physically model particular UAVs, the procedure carried out to assess these system has been very brief and lacks a more detailed analysis of conventional quadrotors with regards to their dynamic behavior.

This research focuses on carrying out an in depth analysis of a conventional quadrotor using the mathematical representation which will be assessed on Simulink. On the other hand, the work will also continue to use ADAMS environment by importing a geometry with identical parameters to the mathematical representation using SolidWorks. By selecting appropriate actuators, propellers and the body frame parameters, the motions of the proposed quadrotor can be analyzed in Simulink without using any means of control strategies. Hence, increasing the speed of individual actuators will generate thrusts based on the aerodynamics loads of the propellers, forcing the vehicle to change in motion. Upon collecting the numerical results from Simulink based on rolling, pitching and the vertical lift, the work progresses into introducing the geometry on ADAMS where thrusts will be considered as control inputs identical to the Simulink model. Since this software contains a solver that is capable of delivering realistic virtual results without the requirement of 
any mathematical equations. Justification of the numerical data attained from Simulink can be compared against the results of ADAMS.

The paper is structured as follows. In section 2, the dynamic model for a full quadrotor is theoretically presented which consists of the Euler's rotational matrix and the full equation of motion for a plus configured quadrotor. In section 3, a quadrotor model is proposed which consists of appropriate parameters that represents the vehicle frame, motor and propeller characteristics. Section 4 presents the simulation results from Simulink and ADAMS where a comparative study between both systems are thoroughly analyzed. Finally, Section 5 concludes the work attained and the overall performance from both simulation tools.

\section{QUADROTOR DYNAMICS}

Quadrotors are commonly constructed in a plus configuration due its simplicity of development and wide selection. As illustrated in figure $1, \omega_{m 1}$ to $\omega_{m 4}$ defines the angular velocity of the respective motor 1 to 4 . In order to achieve lift, motor 1 and 3 are set to rotate clockwise while motor 2 and 4 are set to rotate in the counter clockwise direction. Increasing and decreasing the speed of individual motors will enforce the UAV to achieve motions along the desired axis. That is, if all the motor speeds are simultaneously increased, the vehicle will lift vertically upwards as indicated by the arrows in figure 1 (a). Increasing the speed of motor 2 while keeping the other three constant will enforce the UAV to pitch as shown in figure 1 (b). Similarly, if the angular speed of motor 3 increases while maintaining the other three at a constant speed will enforce the vehicle to roll as shown in figure 1 (c). Finally, increasing the angular velocity of motor 1 and 3 while reducing motor 2 and 4 simultaneously generates a yawing motion.

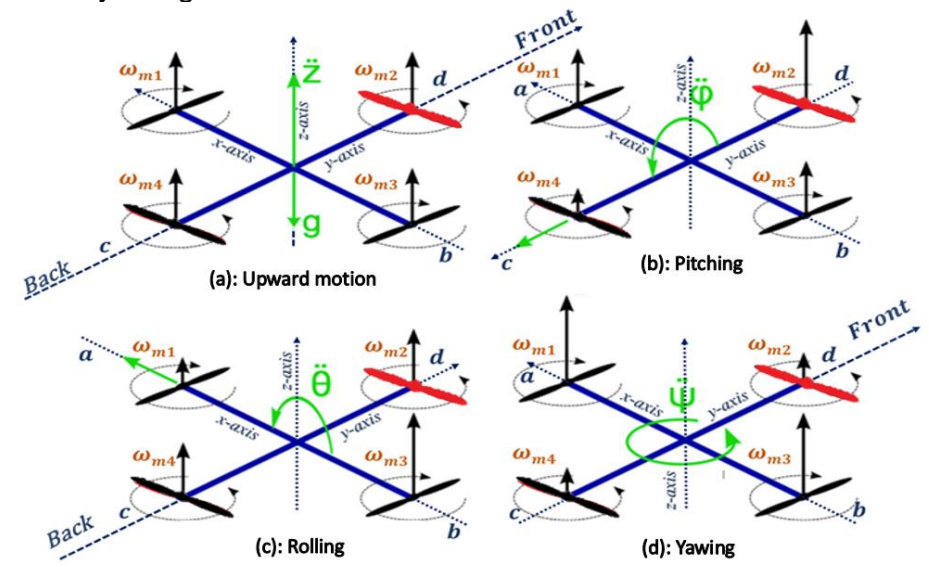

Figure 1: Quadrotor operation for; (a) Altitude, (b) Pitching, (c) Rolling, (d) Yawing

As this work consists of analyzing the quadrotor performance without accompanying any control algorithms, the mathematical description of these motions assumes that the drone is rigid and has a symmetric structure; all propellers are equal in size; all motors are from the same model and are placed at equal distances to the center of mass. Twelve states characterize the translational and rotational behavior of the vehicle body inertial frame with respect to the fixed inertial frame:

$$
x^{T}=\{x, \dot{x}, y, \dot{y}, z, \dot{z}, \phi, \dot{\phi}, \theta, \dot{\theta}, \psi, \dot{\psi})
$$

Where $\{x, y, z, \dot{x}, \dot{y}, \dot{z}\}$ represents the translational position and velocity along the respective axis while $\{\phi, \theta, \psi, \dot{\phi} . \dot{\theta}, \dot{\psi}\}$ signifies the angular position and velocity for rolling, pitching and yawing [14]. The orientations of the Quadrotor is mathematically described using Euler's angles transformation matrix as shown on equation (2).

$$
\mathbb{R}_{M}=\left(\begin{array}{ccc}
c_{\phi} c_{\theta} & c_{\phi} s_{\theta} s_{\psi}-c_{\psi} s_{\phi} & c_{\phi} s_{\theta} c_{\psi}+s_{\phi} s_{\psi} \\
c_{\theta} s_{\phi} & c_{\phi} c_{\psi}+s_{\phi} s_{\theta} s_{\psi} & s_{\phi} s_{\theta} c_{\psi}-c_{\phi} s_{\psi} \\
-s_{\theta} & c_{\theta} s_{\psi} & c_{\theta} c_{\psi}
\end{array}\right)
$$


Where $s$ and $c$ represents the sin and cos. Equation (3) and (4) represents the translational moments as the vehicle rotates and the moment inertia for the corresponding motions which are defined as $r^{b f}$ and $J^{b f}$ [15].

$$
r^{b f}=[x, y, z]
$$

The moment of inertia for the three rotations around $x, y$ and $z$ are described as:

$$
J^{b f}=\left(\begin{array}{ccc}
J_{x} & -J_{x y} & -J_{x z} \\
-J_{x y} & J_{y} & -J_{y z} \\
-J_{x z} & -J_{y z} & J_{z z}
\end{array}\right)
$$

Where for instance, $J_{x y}=\int x y d m$ is the product of inertia and $J_{z z}=\int\left(x^{2}+z^{2}\right) d m$ is the moment of inertia about the $z$-axis for a rigid body.

\subsection{Gyroscopic Effects and Euler Rotations of a Rigid body}

The three rotational equations of motions are derived about the respective axis represented in Equation (3). As the body rotates and translates, any particles of mass will generally experience some acceleration, where the moment about each axis can be described as [16]:

$$
\begin{aligned}
& I_{x} \ddot{\phi}+\dot{\theta} \dot{\psi}\left(I_{z}-I_{y}\right)=\tau_{x} \\
& I_{y} \ddot{\theta}+\dot{\phi} \dot{\psi}\left(I_{x}-I_{z}\right)=\tau_{y} \\
& I_{z} \ddot{\psi}+\dot{\phi} \dot{\theta}\left(I_{y}-I_{x}\right)=\tau_{z}
\end{aligned}
$$

Where $\tau_{x}, \tau_{y}$ and $\tau_{z}$ represents the sum of the rotational moments. $I_{x}, I_{y}$ and $I_{z}$ describes the moments of inertia around the $x, y$ and $z$ axes respectively. As the vehicle begins to rotate, a gyroscopic torque is generated as the angular momentum of the motors changes $[15,17,18]$ :

$$
G_{a}=\sum_{i=1}^{4} J_{r} \dot{\omega} \Omega_{i}
$$

Where $J_{r}$ signifies the motors moment of inertia, $\dot{\omega}$ is the angular velocity of the body frame and $\Omega_{i}$ is the angular rate of the rotor $i(i=1,2,3,4)$.

\subsection{Aerodynamic Effects}

In quadrotors, aerodynamic loads are generated by propellers placed coincident to the motor rods where a thrust is generated as the angular velocity of the corresponding motor increases. This is mathematically described as [19, 20]:

$$
T_{i}=K \Omega_{i}^{2}
$$

Where $T_{i}$ defines the thrust generated for the corresponding brushless DC (BLDC) motor $i(i=1,2,3,4)$. The angular velocity of the BLDC motor is represented by $\Omega_{i}^{2}$ and $K$ is a representation of either the thrust factor $b$ or the drag factor $d$ which are chosen according to the desired orientation of the quadrotor.

$$
\begin{aligned}
& b=C_{T} \rho D^{4} \\
& d=C_{P} \rho D^{5}
\end{aligned}
$$

Where $C_{T}$ and $C_{P}$ are the thrust and drag coefficients, $\rho$ is the air density and $D$ is the propeller diameter. The parameters obtained from [21] represents the propellers characteristics as shown in table 1.

Table 1: Propeller characteristics (10x5.5)

\begin{tabular}{lcc}
\hline \multicolumn{1}{c}{ Parameter } & Symbol & Value \\
\hline Diameter & $D$ & $0.254 \mathrm{~m}$ \\
Thrust coefficient & $C_{T}$ & 0.121 \\
Power Coefficient & $C_{P}$ & 0.0495 \\
Air density & $\rho$ & $1.255 \mathrm{Kg} / \mathrm{m}^{3}$ \\
\hline
\end{tabular}

Based on the values obtained from table 1, the thrust and drag factor are calculated as:

$$
\begin{gathered}
b=C_{T} \rho D^{4}=6.317 \times 10^{-4} \\
d=C_{P} \rho D^{5}=1.61 \times 10^{-4}
\end{gathered}
$$




\subsection{Motor Control Input}

Since this work is focused on analyzing a plus configured quadrotor, the thrust generated by the motors to achieve the desired motion is expressed as the addition and subtraction of the four motors respectively which is denoted as:

$$
\begin{aligned}
U_{1} & =b\left(\Omega_{1}^{2}+\Omega_{2}^{2}+\Omega_{3}^{2}+\Omega_{4}^{2}\right) \\
U_{2} & =b\left(-\Omega_{2}^{2}+\Omega_{4}^{2}\right) \\
U_{3} & =b\left(-\Omega_{1}^{2}+\Omega_{3}^{2}\right) \\
U_{4} & =d\left(-\Omega_{1}^{2}+\Omega_{2}^{2}-\Omega_{3}^{2}+\Omega_{4}^{2}\right)
\end{aligned}
$$

Where $U_{1}$ represents the overall thrust generated by the propellers, $U_{2}, U_{3}$ and $U_{4}$ defines the total thrust to achieve rolling, pitching and yawing respectively. Increasing or decreasing the thrust generation is described as adjusting the angular velocities of independent motors; $\Omega_{1}, \Omega_{2}, \Omega_{3}$ and $\Omega_{4}$. As for the gyroscopic effects presented in equation (8), $\Omega_{r}$ signifies the overall residual propeller angular speed as the vehicle rolls or pitches.

$$
\Omega_{r}=-\Omega_{1}+\Omega_{2}-\Omega_{3}+\Omega_{4}
$$

\subsection{Quadrotor Equation of Motion}

The mathematical model can be described through a combination of equations (5), (6), (7), (8), (9) and (14) in the form of translational and rotational accelerations. The quadrotor mathematical model is obtainable through the Euler equation of motion as:

$$
\begin{aligned}
\ddot{x} & =\frac{1}{m}[\cos \phi \sin \theta \cos \psi-\sin \phi \sin \psi] U_{1} \\
\ddot{y} & =\frac{1}{m}[\cos \phi \sin \theta \sin \psi+\sin \phi \cos \psi] U_{1} \\
\ddot{z} & =-g+\frac{1}{m}[\cos \phi \cos \theta] U_{1} \\
\ddot{\phi} & =\frac{1}{I_{x}}\left[\dot{\theta} \dot{\psi}\left(I_{z}-I_{y}\right)-J_{r} \dot{\theta} \Omega+l U_{2}\right] \\
\ddot{\theta} & =\frac{1}{I_{y}}\left[\dot{\phi} \dot{\psi}\left(I_{x}-I_{z}\right)+J_{r} \dot{\phi} \Omega+l U_{3}\right] \\
\ddot{\psi} & =\frac{1}{I_{z}}\left[\dot{\phi} \dot{\theta}\left(I_{y}-I_{x}\right)+U_{4}\right]
\end{aligned}
$$

Where $m$ denotes the mass of the quadrotor, $g$ denotes the acceleration due to gravity, $l$ represent the length from the rotor to the centre of mass and $J_{r}$ represents the motors moment of inertia.

\section{SYSTEM MODEL}

In order to study the performance of the presented mathematical equations, it is assumed that the vehicle will be assessed in an environment that has negligible air and no external disturbances. The physical parameters selected for this particular study are presented in table 2.

Table 2: Quadrotor Model Parameter Values

\begin{tabular}{cccc}
\hline Variable & Description & Value & Units \\
\hline$g$ & Gravity force & 9.81 & $\mathrm{~ms}^{-2}$ \\
$m$ & Quadrotor mass & 1.888 & $\mathrm{Kg}$ \\
$I_{x}$ & Inertia around x-axis & $1.453 \times 10^{-2}$ & $\mathrm{Kg} \cdot \mathrm{m}^{2}$ \\
$I_{y}$ & Inertia around y-axis & $1.453 \times 10^{-2}$ & $\mathrm{Kg} \cdot \mathrm{m}^{2}$ \\
$I_{z}$ & Inertia around z-axis & $2.884 \times 10^{-2}$ & $\mathrm{Kg} \cdot \mathrm{m}^{2}$ \\
$l$ & Length from rotor to cm & 0.225 & $\mathrm{~m}$ \\
\hline
\end{tabular}

\subsection{Motor Features}

Due to the fact that BLDC motors are manufactured in different sizes, their specifications can greatly vary depending on the operating voltage which effects the overall performance of the vehicle. As for this particular 
research, the motors selected must be capable of lifting the body frame without reaching saturation. Therefore, an ideal BLDC motor that can achieve these requirement is the BN12 model [22] which has the characteristics shown in table 3.

Table 3: BN12 BLDC Motor Parameters

\begin{tabular}{ccc}
\hline Parameter Name & Value & Unit \\
\hline Nominal Voltage & 12 & Volts \\
Rated Speed & 13,027 & $R P M$ \\
No-Load Speed & $\sim 15,900$ & $R P M$ \\
Rotor Inertia & 2.82 & $g-\mathrm{cm}^{2}$ \\
Weight & 102.2 & Grams \\
\hline
\end{tabular}

\subsection{ADAMS Geometry}

Msc ADAMS is an abbreviation for Automatic Dynamic Analysis Mechanical System. It is an extremely powerful software that can analyze and assess almost any mechanical system that consist of multi body interactions [23]. Since ADAMS supports the feature of importing geometries, SolidWorks was used to design and develop the Quadrotor similar to the proposed model. Three main components are designed; the quadrotor body frame, propellers and actuators as shown on figure 2. It is worth mentioning that the dimensions are particularly selected to satisfy the quadrotor model in order to compare the results against Simulink.

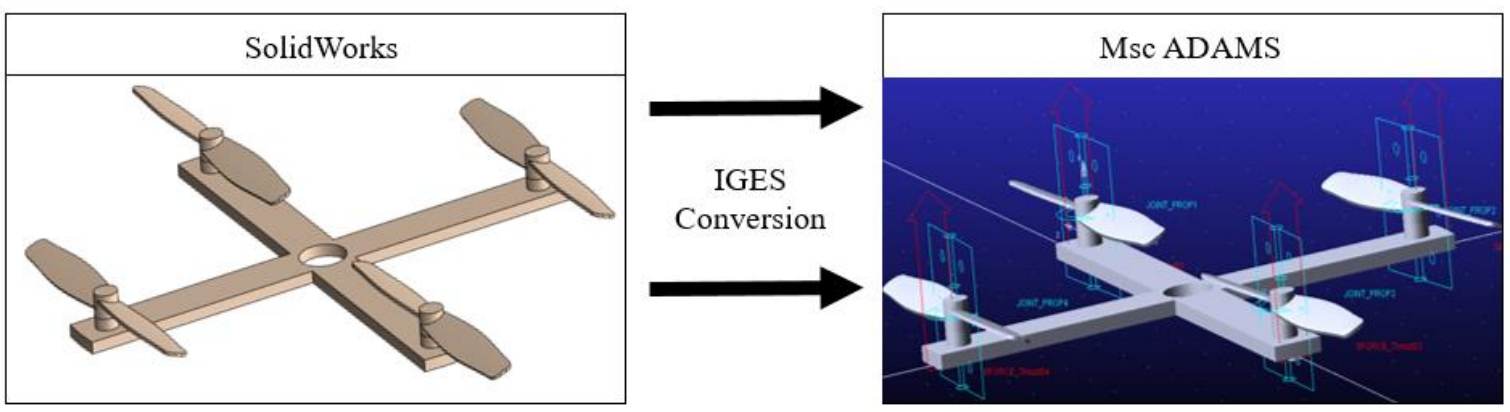

Figure 2: Designed quadrotor on SolidWorks and imported into ADAMS

By importing the designed geometry into ADAMS, the operator defines constraints and joints to the components before carrying out any motion analysis. For this particular study, increasing the angular velocity of individual motors generates a relative thrust where the vehicle motions can be viewed.

In order to obtain simulation results from ADAMS environment, each component within the geometry consists of attributes such as mass, material, moment of inertia, constraints, and drive inputs. Figure 2 also illustrates the imported geometry where the motors are constrained to the quadrotor body using fixed joints while the propellers are also connected to the corresponding motors using rotational joints.

To explain this further, a co-simulation feature is used where the control inputs to the individual motors can be applied from Simulink in a separate study to the mathematical model. Essentially, the angular velocity applied to each motor will generate a corresponding thrust which will enforce the UAV to achieve the desired motion. Defining these quantities on ADAMS is achieved through the inclusion of functions as shown on table 4, where the thrust generated relies on the angular velocity of the respective motors. 
Table 4: Defined attributes and functions in ADAMS

\begin{tabular}{ccc}
\hline Name & Definition & Function \\
\hline$F_{1}$ & SFORCE_Thrust01 & $6.317 \mathrm{e}-4^{*}($ PROPMOTION1*PROPMOTION1) \\
$F_{2}$ & SFORCE_Thrust02 & $6.317 \mathrm{e}-4^{*}($ PROPMOTION2*PROPMOTION2) \\
$F_{3}$ & SFORCE_Thrust03 & $6.317 \mathrm{e}-4^{*}($ PROPMOTION3*PROPMOTION3) \\
$F_{4}$ & SFORCE_Thrust04 & $6.317 \mathrm{e}-4^{*}$ (PROPMOTION4*PROPMOTION4) \\
$M_{1}$ & PROPMOTION1 & VARVAL(SPEED1) \\
$M_{2}$ & PROPMOTION2 & VARVAL(SPEED2) \\
$M_{3}$ & PROPMOTION3 & VARVAL(SPEED3) \\
$M_{4}$ & PROPMOTION4 & VARVAL(SPEED4) \\
\hline
\end{tabular}

Where $F_{i}$ represents the thrust generated by the motor $M_{i}$. VARVAL(.) is a function that considers the angular velocity control inputs; SPEED1-4.

\section{SIMULATION RESULTS AND ANALYSIS}

In this section, the aim is to analyze the dynamic behavior of the vehicle using two simulation tools which are; Simulink that consists of the mathematical model and ADAMS which consists of the quadrotor geometry with identical parameters. Although justification is a main factor considered in this study, having the capability of viewing the performance of the Quadrotor as a virtual model will introduce a platform for researchers such that an improved analysis of UAVs can be carried out, saving costs and time of the physical development. By applying identical control inputs on Simulink and ADAMS, the behavior of the UAV attained from both simulations will be analyzed and compared.

With regards to the six equations of motions presented from equation (16) to (21), the block diagram presented in figure 3 illustrates an overview of the system architecture where the control input corresponds directly to the angular velocity of the corresponding motor. In the control mixer block, equation (14) is considered to generate a relative thrust where the dynamics can be viewed. Finally, applying the double integration to the open loop system will present the respective states of position, velocity and acceleration.

As this paper focuses on justifying the results attained from Simulink, the main point of interest is to ensure that the performances from the virtual software ADAMS and Simulink are matched. Therefore, numerous analysis methods will be carried out which consist of; vertical lift only, vertical lift + Rolling and vertical lift + Pitching. While these motions are considered vital in the flight response of a general quadrotor, the transient behavior from the proposed system can then be further analyzed as the control input is continuously adjusted.

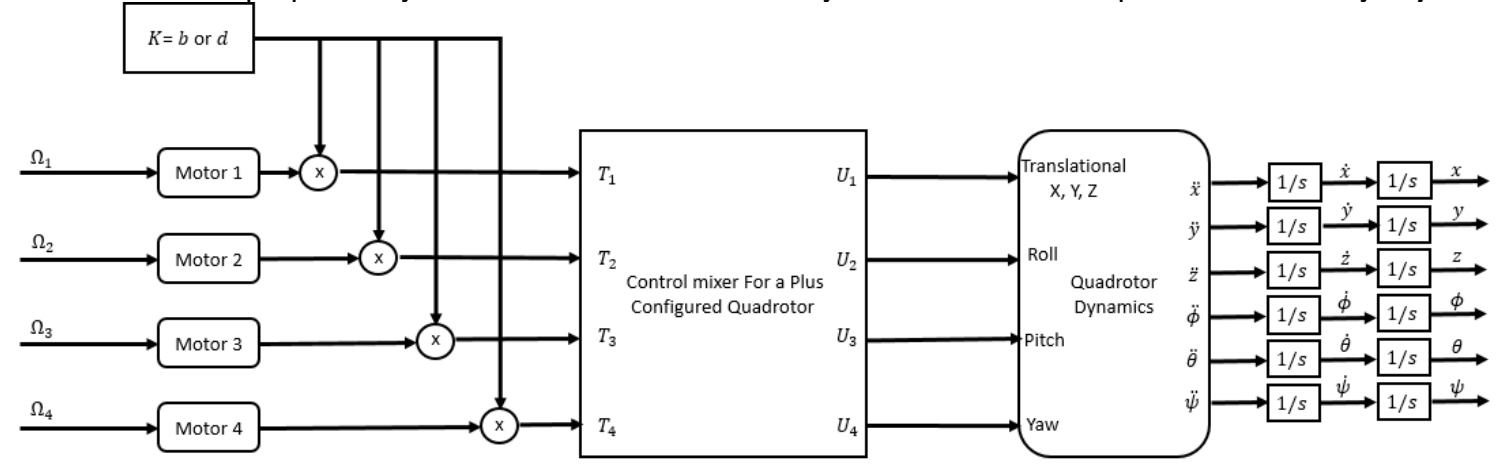

Figure 3: Block Diagram representation of the quadrotor model in Simulink

Since the quadrotor consists of a mass that is approximated to $1.9 \mathrm{Kg}$, the newton's second law states that the thrust required to lift the vehicle must exceed $18.52 \mathrm{~N}$ which is estimated to $4.63 \mathrm{~N}$ (85.61 RPS) per motor. Figure 4 (a) illustrates the transient response of the vehicle during lift as 88.9RPS is simultaneously applied to each motor which has resulted in the vehicle lifting vertically upwards.

As for figure 4 (b), 79.6RPS is simultaneously applied to all the motors where the vehicle began to descend due to the gravitational force overcoming the lift force. In order to ensure that the response between both 
software accurately models the dynamics, the control input for each actuator is set to synchronously oscillate between $0 \mathrm{~N}$ to $15 \mathrm{~N}$ as shown on figure 4 (d). The resultant behavior is seen on figure 4 (c) where the altitude is constantly effected. For instance, as the control input reaches $0 \mathrm{~N}$, the vehicle begins to descend. On the other hand, the vehicle begins to lift as the control input increases towards $15 \mathrm{~N}$. Clearly, the numerical results collected from both software demonstrates a marginal difference between both curves throughout the whole analysis.

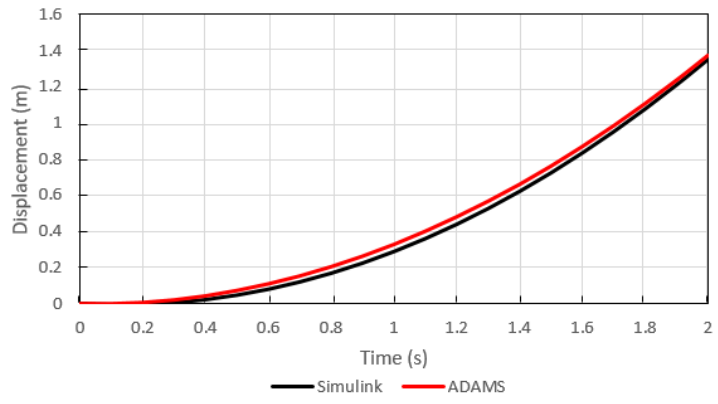

a) Applying $5 \mathrm{~N}$ (88.9RPS) per motor

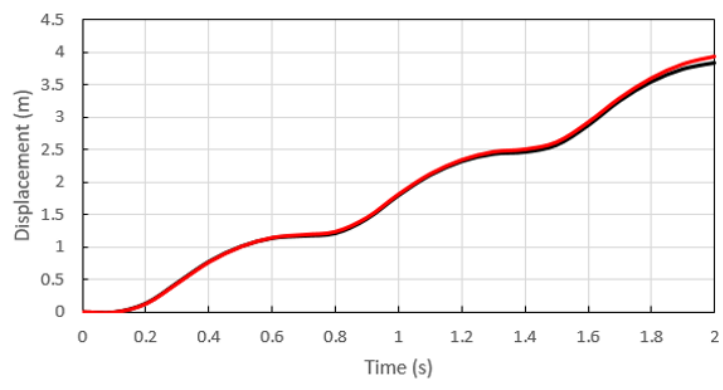

- Simulink - ADAMS

c) Altitude change due to oscillatory control input

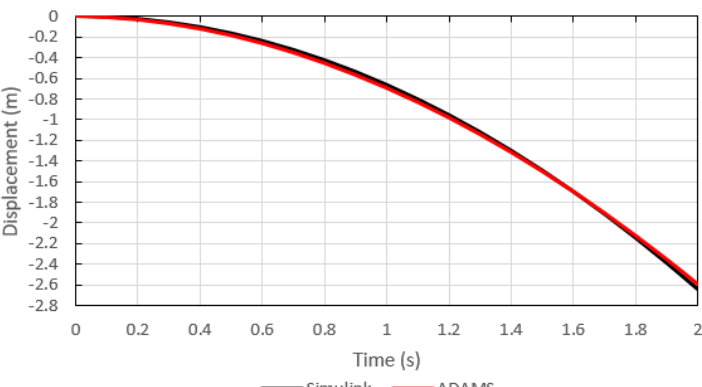

b) Applying $4 \mathrm{~N}$ (79.6RPS) per motor

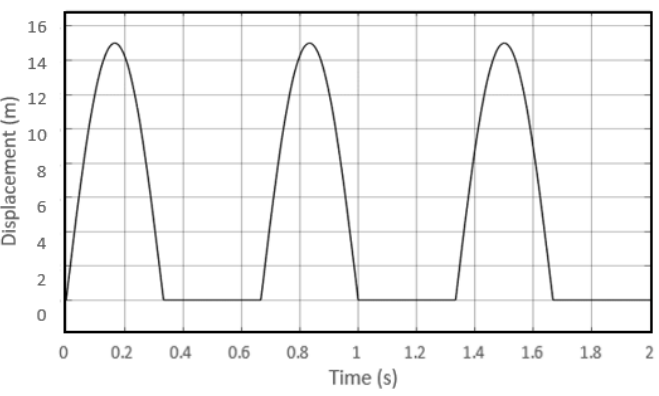

- Control Input (per motor)

d) oscillatory control input between $0-15 \mathrm{~N}$ per motor

Figure 4: Studying the vehicle behavior as the thrusts generated by the motors are simultaneously adjusted.

The study continues to analyze the rotations of rolling and pitching as the angular speed of a single motor is increased while maintaining the others at a constant speed. To assess these rotations, a sinusoidal control input is set to continuously change between $-0.2 \mathrm{~N}$ to $0.2 \mathrm{~N}$ for motor 1 and 2 respectively. Initially, the motors are all fixed to generate a $5 \mathrm{~N}$ thrust which is added to the oscillatory signal shown in figure 5 (e). As a result, the rolling angle is effected as the angular velocity of motor 1 constantly changes between 71.1RPS to 106.7RPS which is shown in figure 5 (a). It is worth mentioning that the rolling angle is coupled with the translations motion for the vehicle along the y-axis. Hence, figure 5 (b) illustrates the translational motion that is achieved due to the variance in the angular velocity of motor 1 .

On the other hand, following an identical approach of continuously changing the angular velocity of motor 2 has presented a superimposed response of the pitching angle similar to the rolling angle as shown on figure 5 (c). Since the pitching angle is coupled with the vehicle translational motions along the x-axis, Figure 5 (d) presents the resultant motions obtained from the quadrotor system which is also superimposed to the translational motions effected by the rolling angle. A comparative analysis based on the motions presented in figure 5 illustrates that there is little to no errors between ADAMS and Simulink results.

It is worth mentioning that although response curves are presented from both software, ADAMS has illustrated a $3 \mathrm{D}$ visual representation of the vehicle during flight. As for Simulink, the motions were only presented as a set of numerical results. 

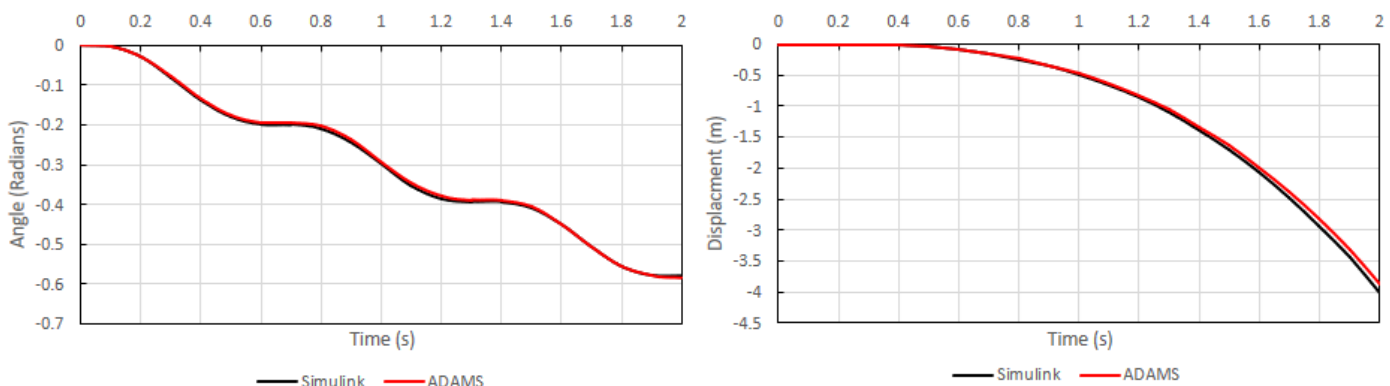

a) Effected $\mathrm{Phi}$ angle due to Motor 1

b) Effected Y-Position due to Motor 1
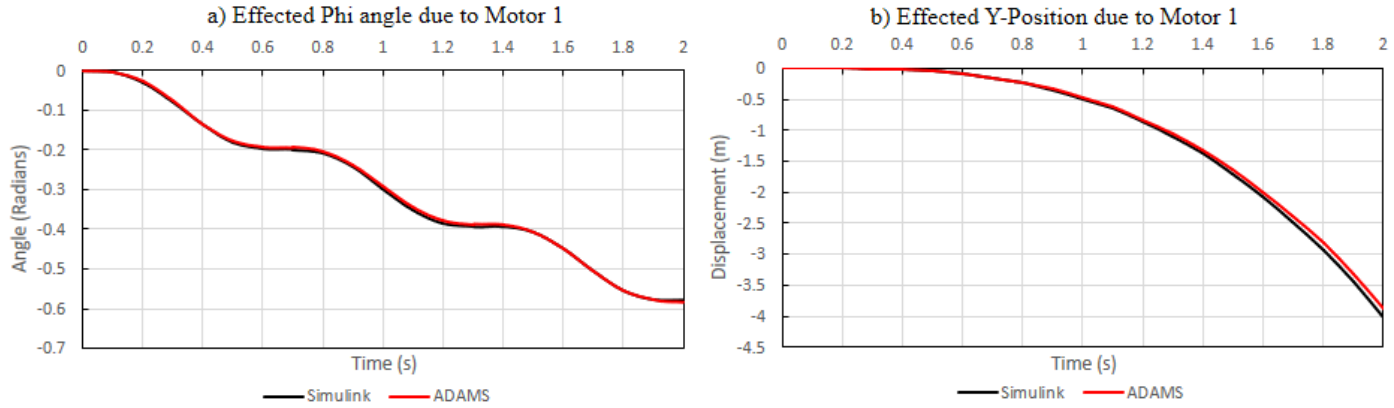

c) Effected Theta angle due to Motor 2

d) Effected X-Position due to Motor 2

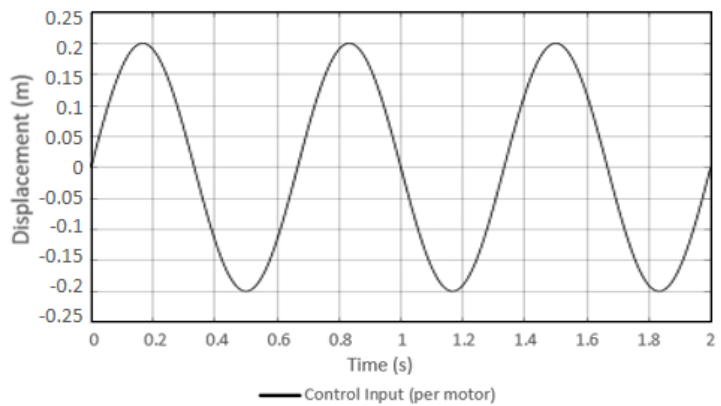

e) Sinusoidal control input changes by $0.2 \mathrm{~N}$ (17.8RPS)

Figure 5: Increasing the angular velocity of motor 1 and 2 to achieve rolling and pitching motions on Simulink and ADAMS.

\section{CONCLUSION}

Currently, a common approach of studying the quadrotor dynamics is simulated using MATLAB/Simulink to verify the flight performance. This paper proposed a method of further analysis and justifications to the current approach, which consists of designing the geometry in SolidWorks followed by using ADAMS for verifications. Based on the studies implemented, the dynamic model has been theoretically presented for all the six degrees of freedom. Appropriate parameters where identified based on previous studies which includes; quadrotor frame, BLDC motor and the propeller characteristics.

From the results attained, it can be concluded that the performance from ADAMS environment has presented similar results to the mathematical model. The software also presented the dynamics as a 3D virtual setting rather than a collection of numerical data from Simulink. However, developing the geometry and running it on ADAMs can be quite complex due to the extensive features that are readily available. Hence, it is vital to ensure that the model is carefully developed and that the dimensions within the geometry are also accurate. Future work will continue to explore ADAMS while using control techniques and modifying the mechanical system. Additionally, carrying out practical developments and comparing the results will also be considered. 


\section{ACKNOWLEDGMENTS}

This research received no grant from any funding agency in the public, commercial, or not-for-profit sectors.

\section{REFERENCES}

[1] E. Chater, H. Housny and H. El Fadil, "Robust Control Design for Quadrotor Trajectory Path Tracking," in In 2019 8th International Conference on Systems and Control (ICSC) (pp. 21-26). IEEE., 2019,.

[2] S. Bansal, A. Akametalu, F. Jiang, F. Laine and C. Tomlin, "Learning quadrotor dynamics using neural network for flight control," in In 2016 IEEE 55th Conference on Decision and Control (CDC) (pp. 4653-4660). IEEE, 2016.

[3] C. Vong, K. Ryan and H. Chung, "Integral Backstepping Position Control for Quadrotors in Tunnel-Like Confined Environments," in In 2019 International Conference on Robotics and Automation (ICRA) (pp. 6425-6431). IEEE, 2019.

[4] Y. Yu and X. Ding, "A global tracking controller for underactuated aerial vehicles: design, analysis, and experimental tests on quadrotor," in IEEE/ASME Transactions on Mechatronics, 21(5), pp.2499-2511, 2016.

[5] A. Salih, M. Moghavvemi, H. Mohamed and K. Gaeid, "Flight PID controller design for a UAV quadrotor.," Scientific research and essays, vol. 5(23), pp. 3660-3667, 2010.

[6] M. Efe, "Robust low altitude behavior control of a quadrotor rotorcraft through sliding modes," in In 2007 Mediterranean Conference on Control \& Automation (pp. 1-6). IEEE, 2007.

[7] E. Zheng, J. Xiong and J. Luo, "Second order sliding mode control for a quadrotor UAV," ISA transactions, vol. 53(4), pp. 1350-1356., 2014.

[8] A. Rusli, M. Aras, M. Aripin, M. Kamarudin, M. Azmi, M. Kasno, A. Khamis and Z. Rizman, "Modelling of unmanned aerial vehicle (UAV) for altitude control using system identification technique.," J Fund Appl Scie, vol. 10, pp. 936-950., 2018.

[9] A. Manecy, N. Marchand, F. Ruffier and S. Viollet, "X4-mag: a low-cost open-source micro-quadrotor and its linux-based controller.," International Journal of Micro Air Vehicles, vol. 7(2), pp. 89-109, 2015.

[10] R. Ryan, "ADAMS—Multibody system analysis software.," In Multibody systems handbook, Vols. Springer, Berlin, Heidelberg., pp. 361 402, 1990.

[11] D. Zhai, X. Jiang and W. Peng, "Co-simulation Research Based on Position Tracking of Quadrotor Vehicles," in In 2018 IEEE International Conference of Intelligent Robotic and Control Engineering (IRCE) (pp. 118-123). IEEE, 2018.

[12] R. Ji and J. Ma, "Mathematical Modeling and Analysis of a Quadrotor with Tilting Propellers," in In 201837 th Chinese Control Conference (CCC) (pp. 1718-1722). IEEE, 2018.

[13] E. Bellocchio, T. Ciarfuglia, F. Crocetti, A. Ficola and P. Valigi, "Modelling and simulation of a quadrotor in V-tail configuration," IJMIC, vol. 26(2), pp. 158-170, 2016.

[14] A. Kamath, V. Tripathi and L. Behera, "Vision-Based Autonomous Control Schemes for Quadrotor Unmanned Aerial Vehicle," in In Aerial Robotic Systems. IntechOpen, 2019.

[15] B. Stevens, F. Lewis and E. Johnson, Aircraft control and simulation: dynamics, controls design, and autonomous systems, John Wiley \& Sons, 2015.

[16] D. Wells, Schaum's Outline of Lagrangian Dynamics: With a Treatment of Euler's Equations of Motion, Hamilton's Equations and Hamilton's Principle, Referenciado en,, 1967

[17] Idrissi, M. and Annaz, F., 2020. Dynamic Modelling and Analysis of a Quadrotor Based on Selected Physical Parameters. International Journal of Mechanical Engineering and Robotics Research, 9(6).

[18] C. Herda, "Implementation of a quadrotor unmanned aerial vehicle," Doctoral dissertation, California State University, Northridge, 2012

[19] A. Zulu and S. John, "A Review of Control Algorithms for Autonomous Quadrotors," Open J. Appl. Science, vol. 04, no. 14, p. 547-556, 2014.

[20] R. Cunha, D. Cabecinhas and C. Silvestre, "Trajectory Tracking tracking control quadrotor vehicle Nonlinear Trajectory Control of of a Quadrotor Vehicle," p. 2763-2768, 2009.

[21] A. Elhennawy, "Dynamic modeling and robust nonlinear control of unmanned quadrotor vehicle.," Doctoral Thesis, 2018.

[22] B. b. D. motor, "Moog," BN12, 2019. [Online]. Available: https://www.moog.com/products/motors-servomotors/brushless-motors/insiderotor-brushless-dc-motors/bn-series/bn12.html. [Accessed 2810 2019].

[23] X. Xue, "Investigation into the Utility of the MSC ADAMS Dynamic Software for Simulating Robots and Mechanisms," Doctoral dissertation, Ohio University, 2013. 


\section{Authors' Research Background}

*The form itself will not be published.

*Title can be chosen from: master student, Ph.D. candidate, assistant professor, lecturer, associate professor, full professor

\begin{tabular}{|c|c|c|c|}
\hline Your Name & Title* & Research Field & Personal website \\
\hline Moad Idrissi & Ph.D. & $\begin{array}{l}\text { Electronic/ } \\
\text { Aerospace }\end{array}$ & $\begin{array}{l}\text { https://www.bcu.ac.uk/research/our- } \\
\text { phds/our-researchers/moad-idrissi }\end{array}$ \\
\hline $\begin{array}{l}\text { Mohammad } \\
\text { Salami }\end{array}$ & $\begin{array}{l}\text { Assistant } \\
\text { Professor }\end{array}$ & Mechanical & $\begin{array}{l}\text { https://www.bcu.ac.uk/engineering/about- } \\
\text { us/our-staff/mohammad-reza-salami }\end{array}$ \\
\hline Fawaz Annaz & $\begin{array}{l}\text { Associate } \\
\text { Professor }\end{array}$ & $\begin{array}{l}\text { Mechatronic/ } \\
\text { Aerospace }\end{array}$ & $\begin{array}{l}\text { https://www.bcu.ac.uk/engineering/about- } \\
\text { us/our-staff/fawaz-annaz }\end{array}$ \\
\hline
\end{tabular}

